\title{
Genetic Variation in Immunoglobulin G Receptor Affects Survival After Lung Transplantation
}

\author{
D. Ruttens ${ }^{1}$, S. E. Verleden ${ }^{1}$, P. C. Goeminne ${ }^{1}$, \\ E. Vandermeulen ${ }^{1}$, E. Wauters ${ }^{2,3}$, B. Cox ${ }^{4}$, \\ R. Vos ${ }^{1}$, D. E. Van Raemdonck ${ }^{1}$, \\ D. Lambrechts ${ }^{2,3}$, B. M. Vanaudenaerde ${ }^{1}$ and \\ G. M. Verleden ${ }^{1, *}$ \\ ${ }^{1}$ Lung Transplant Unit, Laboratory of Pneumology, KU \\ Leuven, University Hospital Gasthuisberg Leuven, \\ Leuven, Belgium \\ ${ }^{2}$ Vesalius Research Centrum (VRC), VIB, Leuven, \\ Belgium \\ ${ }^{3}$ Laboratory for Translational Genetics, Department of \\ Oncology, KU Leuven, Leuven, Belgium \\ ${ }^{4}$ Centre for Environmental Sciences, Hasselt University, \\ Diepenbeek, Belgium \\ *Corresponding author: Geert M. Verleden, \\ geert.verleden@med.kuleuven.be
}

Chronic rejection remains the most important complication after lung transplantation (LTx). There is mounting evidence that both rheumatoid arthritis and chronic rejection share similar inflammatory mechanisms. As genetic variants in the FCGR2A gene that encodes the immunoglobulin gamma receptor (IgGR) have been identified in rheumatoid arthritis, we investigated the relationship between a genetic variant in the IgGR gene and chronic rejection and mortality after LTx. Recipient DNA from blood or explant lung tissue of 418 LTx recipients was evaluated for the IgGR (rs12746613) polymorphism. Multivariate analysis was carried out, correcting for several co-variants. In total, 216 patients had the CC-genotype (52\%), 137 had the CT-genotype $(33 \%)$ and 65 had the TT-genotype $(15 \%)$. Univariate analysis demonstrated higher mortality in the TTgenotype compared with both other genotypes ( $<<0.0001)$. Multivariate analysis showed that the TTgenotype had worse survival compared with the CCgenotype (hazard ratio $[H R]=2.26, p=0.0002$ ) but no significance was observed in the CT-genotype (HR= $1.32, p=0.18)$. No difference was seen for chronic rejection. The TT-genotype demonstrated more respiratory infections (total, $p=0.037$; per patient, $p=0.0022$ ) compared with the other genotypes. A genetic variant in the IgGR is associated with higher mortality and more respiratory infections, although not with increased prevalence of chronic rejection, after LTX.

Keywords: FCGR2A, genetics, IgG, lung transplantation, mortality, respiratory infections

\begin{abstract}
Abbreviations: $A R$, acute rejection; $C F$, cystic fibrosis; $\mathrm{Cl}$, confidence interval; FCGR2A, low-affinity immunoglobulin gamma Fc region receptor II-a; GERD, gastroesophageal reflux disease; HR, hazard ratio; IgG, immunoglobulin gamma; IgGR, immunoglobulin gamma receptor; ILD, interstitial lung disease; ISHLT, International Society for Heart and Lung Transplantation; LB, lymphocytic bronchiolitis; LTx, lung transplantation; PAHT, pulmonary arterial hypertension; SLTx, single lung transplantation; SNP, single nucleotide polymorphism
\end{abstract}

Received 13 November 2013, revised 24 February 2014 and accepted for publication 15 March 2014

\section{Introduction}

Lung transplantation ( $\mathrm{LTX}$ ) is a live-saving treatment option for selected patients suffering from specific end-stage pulmonary disorders. One of the major challenges in the further care of these patients is the considerable rate of chronic rejection (1). Chronic rejection, nowadays known as irreversible chronic lung allograft dysfunction, affects over $50 \%$ of the LTx recipients within 5 years after LTx (2). Chronic rejection is thought to be caused by an abnormal inflammatory response of the small airways, due to several immunological and nonimmunological factors, resulting in a fibroproliferative obliteration of the small airways, causing respiratory insufficiency and eventually death $(3,4)$.

Immunoglobulin gamma $(\mathrm{lgG})$ is a protein representing approximately $75 \%$ of serum immunoglobulins in humans. Recent evidence demonstrates that the number of fungal, bacterial or opportunistic infections is increased in $L T x$ recipients with severe hypogammaglobulinemia $(<4 \mathrm{~g} / \mathrm{L})(5-7)$. IgG levels were also lower in those patients with chronic rejection and in those who subsequently developed chronic rejection compared with patients who were free of chronic rejection, which corresponds with an increased incidence of chronic rejection (6). Severe hypogammaglobulinemia $(<4 \mathrm{~g} / \mathrm{L})$ was associated with a worse 1-year survival rate after $\operatorname{LTx}(7,8)$, possibly caused by an increased number of respiratory infections $(6,8)$. Interestingly, not only post-LTx levels but also baseline (pre-LTX) serum IgG levels seem important for the post-LTX outcome (9). 


\section{IgG Receptor and Outcome After LTx}

A specific genetic variant of the low-affinity IgG Fc region receptor II-a (FCGR2A) is a single nucleotide polymorphism (SNP; rs12746613) $13 \mathrm{~kb}$ away from a missense SNP (nonsynonymous mutation) and is associated with rheumatoid arthritis $(10)$ and lupus erythemathosus $(11,12)$. Both of these diseases have comparable immunological pathways, which are involved in chronic rejection after LTx (13).

In the present study, we postulate that this genetic variant of the IgG receptor (IgGR) (FCGR2A, rs12746613) may influence the long-term outcome of lung transplant patients. We aimed (i) to investigate the association between genetic variants of FCGR2A within our lung transplant cohort and chronic rejection/mortality after LTx and (ii) to assess the association between the selected genetic variant and the number of respiratory infection episodes.

\section{Methods}

\section{Study design}

This study included all patients transplanted between April 1991 and December 2010 in the University Hospital of Leuven. Patient follow-up was recorded until December 2012, resulting in a minimal follow-up of at least 2 years. The study was approved by the Ethics Committee (S54739) of the University Hospital of Leuven, and all living subjects provided written consent prior to study participation. Chronic rejection was defined as a persistent decline of forced expiratory volume in 1 second of at least $20 \%$ compared to the best postoperative value without any other explanation using a computed tomography scan, transbronchial biopsies and bronchoalveolar lavage and cultures, according to the International Society for Heart and Lung Transplantation (ISHLT) criteria (2). Acute rejection (AR) and lymphocytic bronchiolitis (LB) were defined on histopathology according to the ISHLT guidelines (14).

Patient's characteristics included gender, age, time of LTx, type of LTx (single lung transplantation [SLTx] vs. sequential SLTx/heart-lung transplantation) and type of underlying lung disease (emphysema, interstitial lung disease [ILD], pulmonary arterial hypertension [PAHT], cystic fibrosis [CF] and others). Clinical follow-up data included the number of $A R$, LB, gastroesophageal reflux disease (GERD), assessed either by $\mathrm{pH}$ impedance or by gastroscopy within the first 2 years after transplantation (15), date of chronic rejection, use of azithromycin therapy (defined as $250 \mathrm{mg}$ trice weekly for at least three consecutive months) and day of death. Infections were defined as all viral/bacterial/fungal/parasitic respiratory events needing appropriate treatment. Retransplantation $(n=12)$ was considered as a separate transplantation for outcome analyses, since a second allograft was evaluated in an already genotyped recipient, as previously described (16).

\section{Genotyping}

Recipient DNA was extracted from peripheral blood or, when unavailable, from explanted lung tissue during initial transplantation. DNA from blood samples was extracted using the Qiagen QIAamp DNA Blood Midi Kit according to the supplier's instructions (Hilden, Germany). For DNA extraction from lung tissue embedded in paraffin blocks, paraffin was removed by xylene, followed by two washes with ethanol. After the paraffin removal steps, tissue was digested with proteinase $K$ solution and DNA was extracted by using Qiagen DNeasy Blood \& Tissue kit (according to the manufacturer's instructions). For the control of DNA purity, $1 \mu \mathrm{L}$ of genomic DNA was used to measure the $260: 280$ and $260: 230$ ratios by use of a
Nanodrop-1000 (NanoDrop Technologies, Wilmington, DE). Only samples with a 260:280 ratio $<2$ and a 260:230 ratio $<1$ were accepted. DNA (5 ng/ $\mu L)$ was aliquoted into 384-well plates and genotyped at the Vesalius Research Center (Leuven, Belgium).

Genotyping for rs12746613 (10) was performed in a blinded manner using iPLEX technology on a MassARRAY Compact Analyser (Sequenom, Inc., San Diego, CA), as reported previously $(17,18)$. Automated genotyping calls were generated using the MassARRAY RTTM software and were validated by manual review of the raw mass spectra. Quality control was performed by genotyping 12 samples in duplicate, with a duplicate concordance of $100 \%$.

In total, we included 568 patients. DNA extraction failed in 43 patients and, consequently, no DNA was available for genotyping. The genotyping failed in 107 patients, resulting in $418(80 \%)$ successfully genotyped patients.

\section{Statistical analysis}

Graphpad prism 4.0 software (San Diego, CA) was used for statistical analysis of patient's characteristics. Continuous variables are presented as the mean \pm standard error of the mean (SEM), and categorical variables as counts and percentages. Categorical variables are compared between groups using Pearson's chi-squared test, while continuous variables are compared using one-way analysis of variance (ANOVA). The Kruskal-Wallis test, a nonparametric equivalent to the one-way ANOVA, is used for highly skewed variables. Survival rates are calculated with the Kaplan-Meier method and compared between groups by the log-rank test. AR/LB was analyzed as a binary variable comparing ever experiencing one or more $A R /$ LB episodes versus no AR/LB episode during follow-up.

For further statistical analysis, we used SAS software, version 9.3 (SAS Institute, Inc., Cary, NC). Survival analyses were performed to study the effect of the genotype on graft lost (death or graft retransplantation) and chronic rejection. A PROC PHREG statement was used to determine the hazards ratio $(\mathrm{HR})$ for chronic rejection and mortality associated with the genotypes of the genetic variant, while adjusting for age, gender, time of LTx, date of LTx, underlying lung disease, AR, LB and use of azithromycin. By including the time of transplantation and the use of azithromycin in our multivariate analysis, the possible confounding effect of the treatment changes is taken into account. Additionally, in the survival analysis, the time to chronic rejection was included in the model.

\section{Results}

\section{The rs 12746613 population characteristics}

Of the 418 successfully genotyped patients, 216 carried the CC-genotype (52\%), 137 carried the CT-genotype (33\%) and 65 carried the TT-genotype (15\%) (distribution in normal population: CC [64\%]/CT [32\%]/TT [4\%]). No difference was found in the incidence of rheumatoid arthritis in the three genotypes. Patient's characteristics according to the different genotypes are summarized in Table 1. There were no significant differences in gender $(p=0.11)$, age $(p=0.81)$ and type of underlying lung disease $(p=0.093)$ between the three subgroups. Immunosuppressive treatment at Day 90, post-LTx did not differ between the genotypes $(p=0.21$ and $p=0.63$, respectively). The use of azithromycin was comparable in the different genotypes $(p=0.22)$. The presence of GERD was also comparable between the different genotypes $(p=0.70)$. 


\section{Ruttens et al}

Table 1: Characteristics (upper part) and outcome variables (lower part) of our cohort, subdivided according to rs12746613 genotypes

\begin{tabular}{|c|c|c|c|c|}
\hline IgGR polymorphism (FCGR2A) & $\mathrm{CC}$ & CT & $\mathrm{TT}$ & p-Value \\
\hline Number of patients & 216 & 137 & 65 & \\
\hline Female sex & $101(47 \%)$ & $79(58 \%)$ & $30(46 \%)$ & 0.11 \\
\hline Age at LTx (years) & $48.2( \pm 0.9)$ & $48.0( \pm 1.2)$ & $47.6( \pm 1.5)$ & 0.81 \\
\hline Indication for LTx & & & & 0.093 \\
\hline Emphysema, $\alpha-1$ ATD & $102(47 \%)$ & $63(46 \%)$ & $31(48 \%)$ & \\
\hline Pulmonary fibrosis & $32(15 \%)$ & $23(17 \%)$ & $10(15 \%)$ & \\
\hline $\mathrm{CF} /$ bronchiectasis & $37(17 \%)$ & $20(15 \%)$ & $9(14 \%)$ & \\
\hline Eisenmenger/PAHT & $22(10 \%)$ & $10(7 \%)$ & $13(20 \%)$ & \\
\hline Retransplant/others & $23(11 \%)$ & $21(15 \%)$ & $2(3 \%)$ & \\
\hline Type LTx (SS/HLTx, \%) & $179(83 \%)$ & $90(66 \%)$ & $45(69 \%)$ & $0.0007^{*}$ \\
\hline AZI treatment & $125(58 \%)$ & $79(57 \%)$ & $30(46 \%)$ & 0.22 \\
\hline \multicolumn{5}{|l|}{ Treatment at 3 months } \\
\hline FK/CsA & $154(71 \%) / 51(24 \%)$ & $91(67 \%) / 39(28 \%)$ & $37(57 \%) / 21(32 \%)$ & 0.21 \\
\hline AZA/MMF & $154(71 \%) / 24(11 \%)$ & $86(63 \%) / 18(13 \%)$ & $33(51 \%) / 7(11 \%)$ & 0.63 \\
\hline IgGR polymorphism (FCGR2A) & $\mathrm{CC}$ & CT & TT & p-Value \\
\hline Any acute rejection (AR) & $93(43 \%)$ & $65(47 \%)$ & $22(34 \%)$ & 0.19 \\
\hline Severe $A R(\geq A 2)$ & $32(15 \%)$ & $33(24 \%)$ & $12(18 \%)$ & 0.10 \\
\hline Any lymphocytic bronchiolitis (LB) & $75(35 \%)$ & $45(33 \%)$ & $18(28 \%)$ & 0.56 \\
\hline Severe LB ( $\geq B 2)$ & $33(15 \%)$ & $33(24 \%)$ & $10(15 \%)$ & 0.72 \\
\hline GERD & $82(38 \%)$ & $51(37 \%)$ & $21(32 \%)$ & 0.70 \\
\hline Respiratory infections & $78(36 \%)$ & $57(42 \%)$ & $35(54 \%)$ & $0.037^{*}$ \\
\hline Respiratory infection episodes/patient & $0.52( \pm 0.06)$ & $0.69( \pm 0.09)$ & $1.21( \pm 0.19)$ & $0.0022^{*}$ \\
\hline Respiratory infections causing death & $14 / 54(21 \%)$ & $13 / 63(21 \%)$ & $15 / 41(36 \%)$ & 0.20 \\
\hline Time to first respiratory infection (days) & $1221( \pm 161)$ & $1269( \pm 171)$ & $1037( \pm 192)$ & 0.38 \\
\hline Chronic rejection & $63(29 \%)$ & $53(38 \%)$ & $17(26 \%)$ & 0.10 \\
\hline Time to chronic rejection (days) & $1088( \pm 105)$ & $1500( \pm 124)$ & $834( \pm 163)$ & 0.098 \\
\hline Death & $54(25 \%)$ & $63(46 \%)$ & $41(63 \%)$ & $<0.0001^{*}$ \\
\hline Time to death (days) & $928( \pm 119)$ & $1366( \pm 153)$ & $1132( \pm 185)$ & 0.24 \\
\hline Early death ( $<30$ days) & $11(5 \%)$ & $7(5 \%)$ & $7(11 \%)$ & 0.21 \\
\hline Time of follow-up (days) & $1829( \pm 90)$ & $2039( \pm 131)$ & $1949( \pm 205)$ & 0.59 \\
\hline
\end{tabular}

Analysis is performed with a one-way ANOVA or a chi-square. Results are shown in numbers (percentage) or with the mean $\pm \mathrm{SEM}$. A pvalue $<0.05$ was considered significant.

AZA, azathioprine; AZI, azithromycin; CF, cystic fibrosis; CsA, cyclosporine; FK, tacrolimus; GERD, gastroesophageal reflux disease; HLTx, heart-lung transplantation; LTx, lung transplantation; MMF, mycophenolate mophetil; PAHT, pulmonary arterial hypertension; SS, doublesided LTx; $\alpha$-1ATD, alpha-1-antitrypsin deficiency. ${ }^{*} A$ p-value $<0.05$ was considered significant.

Race/ethnicity (up to the second generation) is not an issue in our predominantly Caucasian population (98\%). Type of LTx $(p=0.0007)$ was significantly different in univariate analysis among the different genotypes.

\section{The rs 12746613 variant is associated with mortality after $L T x$}

Unadjusted, a significant difference was identified in mortality rates between rs12746613 genotypes $(p<0.0001$, Figure 1A). The TT-genotype had a 5 -year survival of $52 \%$ (1-year survival 76\%), while the CC-genotype had a 5-year survival of $79 \%$ (1-year survival 92\%). The CT-genotype experienced an intermediary risk with a 5-year survival of $72 \%$ (1-year survival 88\%).

After adjusting for age, gender, date of transplantation, LTX type, diagnosis, AR, LB, respiratory infections, use of azithromycin and chronic rejection, we identified a two times higher risk of mortality in the TT-genotype compared with the CC-genotype $(\mathrm{HR}=2.26,95 \% \mathrm{Cl}$ : [1.47-3.47], $\mathrm{p}=0.0002$ ) (Table 2). No significant effect on mortality comparing the TT-genotype versus CT-genotype was observed ( $\mathrm{HR}=1.32,95 \% \mathrm{Cl}$ : [0.87-2.02], $\mathrm{p}=0.18)$. Multivariate analysis excluding retransplantations $(n=12$ : CC-genotype, $\mathrm{n}=3$; CT-genotype, $\mathrm{n}=7$; TT-genotype, $\mathrm{n}=2$ ) showed similar results.

In multivariate analysis, we found, besides our genetic variant, also other risk factors for mortality such as respiratory infections, time to chronic rejection, SLTx the use of azithromycin (Table 2).

\section{The rs12746613 variant is not associated with chronic rejection after $L T x$}

Using unadjusted analysis, the incidence of chronic rejection was comparable in the three rs12746613 genotypes ( $p=0.29$ ) (Figure 1B). Univariate analysis revealed a tendency for an earlier onset of chronic rejection in the TTgenotype $(p=0.098)$. 
IgG Receptor and Outcome After LTx
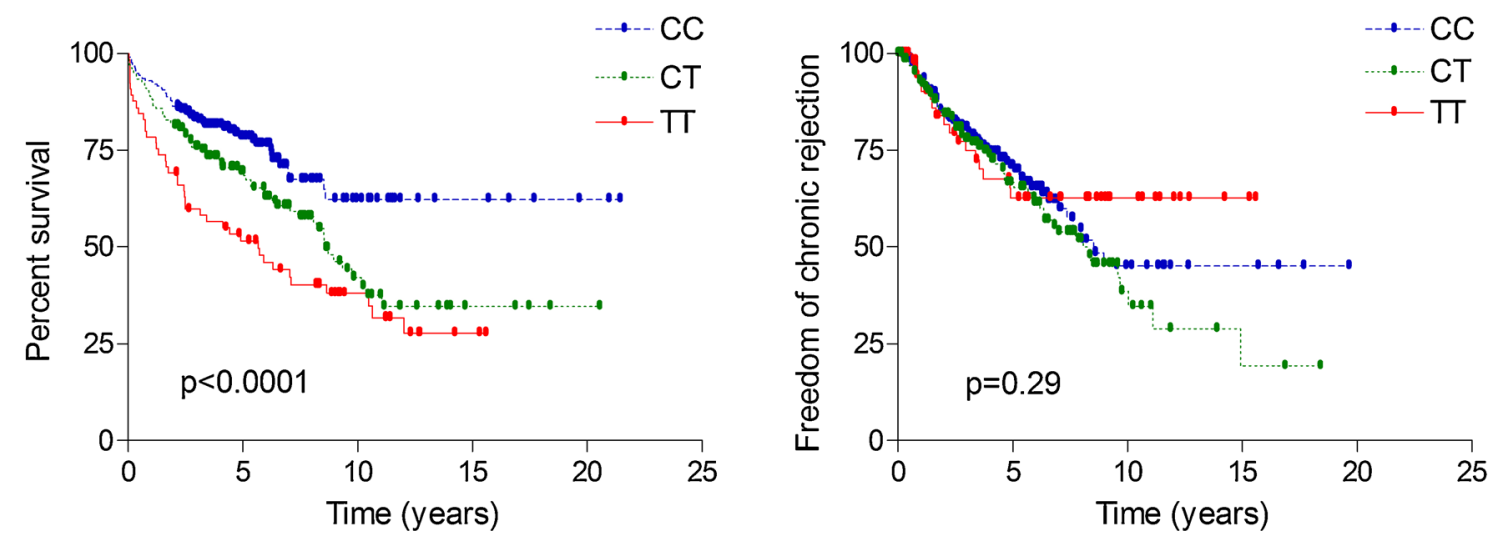

Figure 1: Freedom from mortality (A) and chronic rejection (CR; B) according to rs12746613 genotypes. (A) Unadjusted Kaplan-Meier survival curves in LTx patients classified according to genotype: CC $(n=216), C T(n=137)$ and TT $(n=65)$. TT-genotype has a significant higher risk for mortality $(p<0.0001)$. At the bottom of each graph, the number of patients remaining at risk (by genotype) at various time points posttransplantation is provided. (B) Unadjusted Kaplan-Meier curves of chronic rejection in LTx patients classified according to genotype: CC $(n=216), C T(n=137)$ and TT $(n=65)$. TT-genotype has a similar risk for the development from chronic rejection $(p=0.29)$. LTx, lung transplantation. At the bottom of each graph, the number of patients remaining at risk (by genotype) at various time points posttransplantation is provided.

Multivariate analysis identified a similar risk for chronic rejection for the TT-genotype compared with the CCgenotype ( $\mathrm{HR}=0.71,95 \% \mathrm{Cl}:[0.40-1.20], \quad \mathrm{p}=0.19)$ (Table 3$)$ and the CT-genotype ( $\mathrm{HR}=0.72,95 \% \mathrm{Cl}$ : $[0.41-$ 1.27], $p=0.25$ ) (Table 3).

Table 2: Multivariate analysis of mortality for the rs 12746613 genotypes

\begin{tabular}{lccc}
\hline Mortality & HR & Confidence interval & p-Value \\
\hline SNP TT versus CC & 2.26 & {$[1.47-3.47]$} & 0.0002 \\
SNP TT versus CT & 1.32 & {$[0.87-2.02]$} & 0.18 \\
Resp infection & 1.42 & {$[1.01-2.01]$} & 0.04 \\
AR & 0.83 & {$[0.59-1.17]$} & 0.31 \\
LB & 0.63 & {$[0.44-1.01]$} & 0.056 \\
Age & 1.01 & {$[0.99-1.02]$} & 0.62 \\
Gender & 1.13 & {$[0.81-1.58]$} & 0.48 \\
Diagnosis 0 vs. 4 & 0.64 & {$[0.37-1.09]$} & 0.10 \\
Diagnosis 1 vs. 4 & 0.86 & {$[0.48-1.54]$} & 0.61 \\
Diagnosis 2 vs. 4 & 0.71 & {$[0.35-1.46]$} & 0.35 \\
Diagnosis 3 vs. 4 & 1.10 & {$[0.57-2.16]$} & 0.75 \\
Azithromycin & 2.85 & {$[1.95-4.17]$} & $<0.0001$ \\
Date LTx & 0.82 & {$[0.54-1.23]$} & 0.33 \\
Type LTx & 1.59 & {$[1.05-2.42]$} & 0.027 \\
Time to chronic rejection & 9.59 & {$[6.38-14.42]$} & $<0.0001$ \\
\hline Using a regression mode
\end{tabular}

Using a regression model with corrections for age, gender, LTX type, diagnosis, time of transplantation, A-grade, lymphocytic bronchiolitis (LB), respiratory infections, the use of azithromycin and the time to chronic rejection. Hazard ratio (HR) and 95\% confidence interval are used. A $p$-value $<0.05$ was considered significant.

$A R$, acute vascular rejection; Resp, respiratory; SNP, single nucleotide polymorphism; LTX, lung transplantation.

0-emphysema; 1—interstitial pulmonary fibrosis; 2-cystic fibrosis; 3-pulmonary arterial hypertension; 4-others.
In the multivariate analysis, we found other risk factors for chronic rejection including respiratory infections, AR and the use of azithromycin. Underlying emphysema, CF and PAHT were protective for the development of chronic rejection, compared to ILD and "other" group (Table 3).

Table 3: Multivariate analysis of chronic rejection for the rs12746613 genotypes (upper part)

\begin{tabular}{lccc}
\hline Chronic rejection & HR & Confidence interval & p-Value \\
\hline SNP TT versus CC & 0.71 & {$[0.40-1.20]$} & 0.19 \\
SNP TT versus CT & 0.72 & {$[0.41-1.27]$} & 0.25 \\
Resp infection & 3.23 & {$[2.22-4.76]$} & $<0.0001$ \\
AR & 1.72 & {$[1.20-2.50]$} & 0.0028 \\
LB & 1.05 & {$[0.72-1.54]$} & 0.78 \\
Age & 0.99 & {$[0.98-1.02]$} & 0.84 \\
Gender & 0.84 & {$[0.59-1.21]$} & 0.35 \\
Diagnosis 0 vs. 4 & 0.43 & {$[0.23-0.80]$} & 0.0076 \\
Diagnosis 1 vs. 4 & 0.88 & {$[0.46-1.70]$} & 0.70 \\
Diagnosis 2 vs. 4 & 0.51 & {$[0.27-0.98]$} & 0.045 \\
Diagnosis 3 vs. 4 & 0.44 & {$[0.21-0.93]$} & 0.032 \\
Azithromycin & 3.70 & {$[2.17-6.25]$} & $<0.0001$ \\
Date LTx & 0.96 & {$[0.61-1.55]$} & 0.86 \\
Type LTx & 1.00 & {$[1.00-1.00]$} & 0.74 \\
\hline
\end{tabular}

Using a regression model with corrections for age, gender, LTx type, diagnosis, time of transplantation, A-grade, lymphocytic bronchiolitis (LB), respiratory infections and the use of azithromycin. Hazard ratio (HR) and 95\% confidence interval are used. A pvalue $<0.05$ was considered significant.

AR, acute vascular rejection; Resp, respiratory; SNP, single nucleotide polymorphism; LTx, lung transplantation.

0-emphysema; 1-interstitial pulmonary fibrosis; 2-cystic fibrosis; 3-pulmonary arterial hypertension; 4-others. 


\section{Ruttens et al}

\section{Association between rs 12746613 and respiratory infections}

The TT-genotype seemed to be more prone to develop respiratory infections, 35/65 (54\%) of the patients with the TT-genotype experienced one or more respiratory infections during the posttransplant follow-up. Fifty-seven of 137 patients (42\%) in the CT-genotype group and 78/ 216 patients $(36 \%)$ in the CC-genotype developed one or more respiratory infections $(p=0.037)$ (Table 1$)$. The time to follow-up in the three groups was comparable $(p=0.59)$ (Table 1). Not only the number of patients with respiratory infections was higher in the TT-genotype but also the number of respiratory infections per patient (mean of 1.21/ per patient) compared to the CC-genotype (mean of 0.52 / per patient), with an intermediary number of respiratory infections per patient in the CT-genotype (mean of 0.69/per patient) $(p=0.0022)$. The time to first respiratory infection was not significantly different between the genotypes $(p=0.38)$. Respiratory infections as cause of death were not different in the three genotypes $(p=0.20)$.

\section{Discussion}

Our analysis suggests a role of a genetic variant (rs12746613) in the Fc part of the IgGR gene in mortality and respiratory infections after LTX. Patients with TTgenotype had two times more chance to die during followup compared to the CC-genotype $(p=0.0002)$, while patients with CT-genotype demonstrated an intermediary risk $(p=0.18)$. No association with chronic rejection was found. A clear association was found with respiratory infections, both the total number of patients with one or more respiratory infections $(p=0.037)$ and the number of respiratory infections per patient $(p=0.0022)$. These results suggest that this genetic variant may functionally affect the $\operatorname{lgGR}$ and thereby contribute to mortality and respiratory infections after LTX.

An Fc receptor (CD 32) is a protein found on the surface of certain cells-including B-lymphocytes, natural killer cells, macrophages, neutrophils and mast cells-that contributes to the protective function of the immune system. The Fc receptor binds to antibodies that are attached to infected cells or invading pathogens, which may lead to prevention of respiratory infections. As a consequence, we speculated that a genetic variant in the Fc receptor may be associated with a change in respiratory infections, which is confirmed in the present study.

Recently, some studies described the possible importance of IgG after LTx, since not only the incidence of respiratory infections but also the outcome (chronic rejection/mortality) seems to be influenced by lgG levels (7). A recently published meta-analysis demonstrated an association between hypogammaglobulinemia and increased 1-year mortality in solid organ transplantations (8). We now demonstrated for the first time that a genetic variation in the IgGR gene (rs12746613 SNP [1q23.3] in FCGR2A) may also affect post-LTx mortality, as we established a direct effect on the 1-year survival of the TT-genotype (76\%), compared to CC- and CT-genotype (respectively, 92\% and $88 \%$ survival).

We believe that our findings are further strengthened by the demonstration of a possible functional impact of the investigated FCGR2A genetic variant, as there is a clear association with an increased rate and number of respiratory infections. We could not find an association between respiratory infections and the cause of death, possibly due to the small numbers. However, in multivariate analysis, respiratory infections were a risk factor for mortality.

Unfortunately, we could not demonstrate a direct effect on pre/post-LTx IgG levels, as IgG levels are not routinely measured in our center (only in $234,56 \%$ of our patients, measurements of pre-LTx IgG levels were available). The lack of data is due to the fact that in Belgium, no reimbursement of intravenous immune globulin treatment is available in patients with low lgG levels who are on chronic corticosteroid therapy (an important number of the pretransplant patients); therefore, the determination of IgG levels had no therapeutic consequence. Another concern of this study and possible explanation for the lack of correlation to lgG levels is the fact that no data in literature have suggested that this genetic polymorphism of FCGR2A affects antibody levels or frequency of infection by modulation of IgG levels. Possibly other mechanisms play a role like the regulation of dendritic cell, natural killer or B cell function, which can clearly affect the response to infections or immune regulation. Although this is a very interesting hypothesis, this was beyond the scope of the present study.

In our study, we included 418 patients, making this one of the largest cohorts used for a genetic study in the field of LTX. The single-center approach may result in a more standardized and uniform care, which reduces possible bias by therapy. We are well aware that the lack of a replication cohort is a major concern, but finding a similar cohort of this size and with matched patient data was unfortunately not possible.

In conclusion, we showed that certain genetic variations in the $\operatorname{lgG}$ gene are linked with an increase in respiratory infections and mortality. Our findings undeniably result in further research questions. It not only remains to be established whether regular IgG measurement before and after LTx poses any benefits but also suggests that immunoglobulin substitution in LTx patients with low lgG levels may alter infection rate, but more importantly survival.

\section{Acknowledgment}

David Ruttens takes responsibility for the content of the manuscript, including the data and analysis. 


\section{IgG Receptor and Outcome After LTx}

\section{Author contributions}

DR: Concept and design of the design, drafting manuscript. SEV: Participated in data acquisition and drafting the manuscript. PCG: Participated in statistical analysis and revising the manuscript. EV: Participated in data acquisition and revising the manuscript. EW: Participated in data acquisition and revising the manuscript. BC: Participated in statistical analysis and revising the manuscript. RV: Design of the study and drafting the manuscript. DEV: Participated in data analysis and critical revision. DL: Concept of the study and critical revision. BMV: Concept of the study and critical revision. GMV: Concept and design of the study and critical revision. GMV is holder of a Glaxo Smith Kline (Belgium) chair in respiratory pharmacology at the $\mathrm{KU}$ Leuven; grants from the Research Foundation Flanders (FWO): G.0723.10, G.0753.10, G.0679.12 and G.0705.12; grant from the KU Leuven: OT10/050. EW is junior fellow of the FWO. SEV is funded by the research fund KU Leuven.

\section{Disclosure}

The authors of this manuscript have no conflicts of interest to disclose as described by the American Journal of Transplantation.

\section{References}

1. Verleden GM. Chronic allograft rejection (obliterative bronchiolitis). Semin Respir Crit Care Med 2001; 22: 551-558.

2. Estenne M, Maurer JR, Boehler A, et al. Bronchiolitis obliterans syndrome 2001: An update of the diagnostic criteria. J Heart Lung Transplant 2002; 21: 297-310.

3. Vanaudenaerde BM, Verleden SE, Vos R, et al. Innate and adaptive interleukin-17-producing lymphocytes in chronic inflammatory lung disorders. Am J Respir Crit Care Med 2011; 183: 977-986.

4. Boehler A, Kesten S, Weder W, Speich R. Bronchiolitis obliterans after lung transplantation: A review. Chest 1998; 114: 1411-1426.

5. Bhaskaran A, Hosseini-Moghaddam SM, Rotstein C, Husain S. Mold infections in lung transplant recipients. Semin Respir Crit Care Med 2013; 34: 371-379.
6. Chambers DC, Davies B, Mathews A, Yerkovich ST, Hopkins PM. Bronchiolitis obliterans syndrome, hypogammaglobulinemia, and infectious complications of lung transplantation. J Heart Lung Transplant 2013; 32: 36-43.

7. Kawut SM, Shah L, Wilt JS, et al. Risk factors and outcomes of hypogammaglobulinemia after lung transplantation. Transplantation 2005; 79: 1723-1726.

8. Florescu DF, Kalil AC, Qiu F, Schmidt CM, Sandkovsky U. What is the impact of hypogammaglobulinemia on the rate of infections and survival in solid organ transplantation? A meta-analysis. Am J Transplant 2013; 13: 2601-2610.

9. Yip NH, Lederer DJ, Kawut SM, et al. Immunoglobulin G levels before and after lung transplantation. Am J Respir Crit Care Med 2006; 173: 917-921.

10. Raychaudhuri S, Thomson BP, Remmers EF, et al. Genetic variants at CD28, PRDM1 and CD2/CD58 are associated with rheumatoid arthritis risk. Nat Genet 2009; 41: 1313-1318.

11. Duits AJ, Bootsma $H$, Derksen $R H$, et al. Skewed distribution of IgG Fc receptor Ila (CD32) polymorphism is associated with renal disease in systemic lupus erythematosus patients. Arthritis Rheum 1995; 38: 1832-1836.

12. Harley JB, Alarcon-Riquelme ME, Criswell LA, et al. Genome-wide association scan in women with systemic lupus erythematosus identifies susceptibility variants in ITGAM, PXK, KIAA1542 and other loci. Nat Genet 2008; 40: 204-210.

13. Ziolkowska M, Koc A, Luszczykiewicz G, et al. High levels of IL-17 in rheumatoid arthritis patients: IL-15 triggers in vitro IL-17 production via cyclosporin A-sensitive mechanism. J Immunol 2000; 164: 2832-2838.

14. Stewart S, Fishbein MC, Snell Gl, et al. Revision of the 1996 working formulation for the standardization of nomenclature in the diagnosis of lung rejection. J Heart Lung Transplant 2007; 26: 1229-1242.

15. Vos R, Vanaudenaerde BM, Ottevaere A, et al. Long-term azithromycin therapy for bronchiolitis obliterans syndrome: Divide and conquer? J Heart Lung Transplant 2010; 29: 1358-1368.

16. Nawrot TS, Vos R, Jacobs $L$, et al. The impact of traffic air pollution on bronchiolitis obliterans syndrome and mortality after lung transplantation. Thorax 2011; 66: 748-754.

17. Wauters E, Smeets D, Coolen J, et al. The TERT-CLPTM1L locus for lung cancer predisposes to bronchial obstruction and emphysema. Eur Respir J 2011; 38: 924-931.

18. Lambrechts D, Buysschaert I, Zanen P, et al. The 15q24/25 susceptibility variant for lung cancer and chronic obstructive pulmonary disease is associated with emphysema. Am J Respir Crit Care Med 2010; 181: 486-493. 